\title{
Asymptotics of Extremal Controls in the Sub-Riemannian Problem on the Group of Motions of Euclidean Space
}

\author{
A. P. Mashtakov, A. Yu. Popov
}

We consider a sub-Riemannian problem on the group of motions of three-dimensional space. Such a problem is encountered in the analysis of 3D images as well as in describing the motion of a solid body in a fluid. Mathematically, this problem reduces to solving a Hamiltonian system the vertical part of which is a system of six differential equations with unknown functions $u_{1}, \ldots, u_{6}$. The optimality consideration arising from the Pontryagin maximum principle implies that the last component of the vector control $\bar{u}$, denoted by $u_{6}$, must be constant. In the problem of the motion of a solid body in a fluid, this means that the fluid flow has a unique velocity potential, i.e., is vortex-free. The case $\left(u_{6}=0\right)$, which is the most important for applications and at the same time the simplest, was rigorously studied by the authors in 2017. There, a solution to the system was found in explicit form. Namely, the extremal controls $u_{1}, \ldots, u_{5}$ were expressed in terms of elliptic functions. Now we consider the general case: $u_{6}$ is an arbitrary constant. In this case, we obtain a solution to the system in an operator form. Although the explicit form of the extremal controls does not follow directly from these formulas (their calculation requires the inversion of some nontrivial operator), it allows us to construct an approximate analytical solution for a small parameter $u_{6}$. Computer simulation shows a good agreement between the constructed analytical approximations and the solutions computed via numerical integration of the system.

Keywords: Hamiltonian system, Pontryagin maximum principle, sub-Riemannian, Lie group

Received October 19, 2019

Accepted December 12, 2019

This work was supported by the Russian Science Foundation under grant 17-11-01387 and performed at the Ailamazyan Program Systems Institute of the Russian Academy of Sciences.

Alexey P. Mashtakov

alexey.mashtakov@gmail.com

Anton Yu. Popov

e4e5f4@mail.ru

Ailamazyan Program Systems Institute of RAS

Pereslavl-Zalessky, Yaroslavl Region, 152020 Russia

RUSSIAN JOURNAL OF NONLINEAR DYNAMICS, 2020, 16(1), 195-208 


\section{Introduction}

In the mathematical formalization of control problems for aircraft and floating robots, a subRiemannian (SR) problem arises on the Lie group $\mathrm{SE}_{3}$ of Euclidean motions of three-dimensional space $[1,2]$. SR problems also occur in studies related to image processing. For example, the $\mathrm{SR}$ problem in $\mathrm{SE}_{3}$ appeared in [3], where the authors aim to detect the nerve fibers and blood vessels in three-dimensional MRI (magnetic resonance imaging) images of the human brain.

The SR problem on the Lie group $\mathrm{SE}_{3}$ of Euclidean motions of space $\mathbb{R}^{3}$ can be seen as follows. By given two orthonormal frames $N_{0}=\left\{v_{0}^{1}, v_{0}^{2}, v_{0}^{3}\right\}$ and $N_{1}=\left\{v_{1}^{1}, v_{1}^{2}, v_{1}^{3}\right\}$ attached, respectively, at two given points $q_{0}=\left(x_{0}, y_{0}, z_{0}\right)$ and $q_{1}=\left(x_{1}, y_{1}, z_{1}\right)$ in space $\mathbb{R}^{3}$, the goal is to find the optimal motion of $\mathbb{R}^{3}$ that transfers $q_{0}$ to $q_{1}$ such that the frame $N_{0}$ is transferred to the frame $N_{1}$. The frame can move forward or backward along one of the vectors chosen in the frame and simultaneously rotate around the remaining two (of three) vectors. The required motion should be optimal in the sense of minimal length in the space $\mathrm{SE}_{3} \cong \mathbb{R}^{3} \times \mathrm{SO}_{3}$. See Fig. 1 .

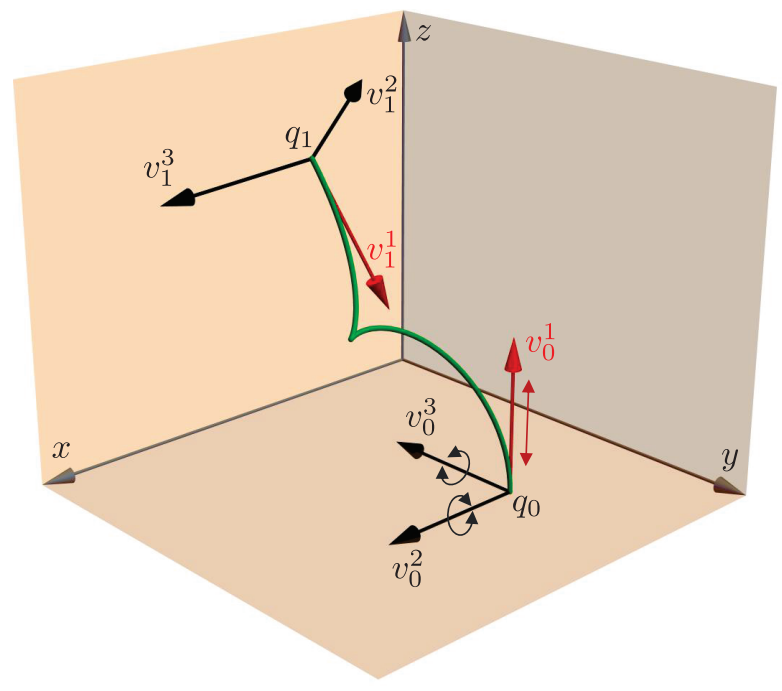

Fig. 1. Statement of the Sub-Riemannian Problem on $\mathrm{SE}_{3}$.

Equivalently, the problem is to find a Lipschitzian curve $\gamma:\left[0, t_{1}\right] \rightarrow \mathrm{SE}_{3}$ such that

$$
\begin{gathered}
\dot{\gamma}=u_{3} \mathcal{A}_{3}+u_{4} \mathcal{A}_{4}+u_{5} \mathcal{A}_{5} \\
\gamma(0)=e, \quad \gamma\left(t_{1}\right)=g \\
l(\gamma)=\int_{0}^{t_{1}} \sqrt{\xi^{2} u_{3}^{2}+u_{4}^{2}+u_{5}^{2}} \mathrm{~d} t \rightarrow \min ,
\end{gathered}
$$

where $\mathcal{A}_{i}$ are left-invariant vector fields on the group $\mathrm{SE}_{3}$, the controls $u_{3}, u_{4}, u_{5}$ are real-valued $\mathbb{L}^{\infty}\left(0, t_{1}\right)$ functions, the terminal time $t_{1}>0$ is free, $e$ is the identity transformation of $\mathbb{R}^{3}, g$ is a given element of $\mathrm{SE}_{3}$ and $\xi>0$ is a parameter that balances the influence of translation and rotations in $\mathbb{R}^{3}$ on the length of the corresponding trajectory.

Investigation of this problem was initiated in $[7,12]$, where in particular it was shown that due to the scaling homothety, see [7, Remark 5], the general case $\xi>0$ reduces to the case $\xi=1$ by linear change of variables. Further in the article, we assume $\xi=1$ without loss of generality. 
The necessary condition of optimality is given by the Pontryagin maximum principle (PMP). Application of PMP to our problem gives an expression of extremal controls in terms of momenta (conjugate variables). Namely, the extremal controls $u_{3}, u_{4}, u_{5}$ coincide with three certain momenta, see [7, Section 3.1]. We denote the remaining three momenta by $u_{1}, u_{2}$ and $u_{6}$. Further, being a little informal, we call the extremal control the entire vector $\bar{u}=\left(u_{1}, u_{2}, u_{3}, u_{4}, u_{5}, u_{6}\right)$ of the momenta. Application of PMP leads to a Hamiltonian system, the horizontal part of which is given by system (1.1) and determines SR geodesics, and the vertical part (on extremal controls) has the following form [7, Eq. (3.5)]:

$$
\left\{\begin{array}{l}
\dot{u}_{1}=-u_{3} u_{5}, \\
\dot{u}_{2}=u_{3} u_{4}, \\
\dot{u}_{3}=u_{1} u_{5}-u_{2} u_{4}, \\
\dot{u}_{4}=u_{2} u_{3}-u_{5} u_{6}, \\
\dot{u}_{5}=u_{4} u_{6}-u_{1} u_{3}, \\
\dot{u}_{6}=0 .
\end{array}\right.
$$

Finding a parameterization of SR geodesics is a nontrivial problem. First, a natural question arises as to the theoretical possibility of such parameterization in some reasonable sense - the question of integrability of the Hamiltonian system, see, e.g., [4-6]. It was shown in [7, Theorem 2] that (1.1)-(1.2) is Liouville integrable, since it has a complete set of functionally independent first integrals in involution. This article is devoted to the study of the vertical part (1.2). It is known that the vertical part has the following first integrals: $u_{6}$, the Hamiltonian $H=\frac{1}{2}\left(u_{3}^{2}+u_{4}^{2}+u_{5}^{2}\right)$ and two Casimir functions $W=u_{1} u_{4}+u_{2} u_{5}+u_{3} u_{6}, C=u_{1}^{2}+u_{2}^{2}+u_{3}^{2}$.

The system of equations (1.2) describes the motion of a solid body in a fluid [8]. In the general case, equations of motion were obtained and studied by G. Kirchhoff [9]. If we consider the mechanical body-fluid system [10] and denote by $\gamma=\left(u_{1}, u_{2}, u_{3}\right)$ the impulse of this system, by $M=\left(u_{4}, u_{5}, u_{6}\right)$ the angular momentum, and by $H=\frac{u_{3}^{2}+u_{4}^{2}+u_{5}^{2}}{2}$ the Hamiltonian which is the kinetic energy of the system, then the Kirchhoff equation

$$
\left\{\begin{array}{l}
\dot{M}=M \times \frac{\partial H}{\partial M}+\gamma \times \frac{\partial H}{\partial \gamma}, \\
\dot{\gamma}=\gamma \times \frac{\partial H}{\partial M}
\end{array}\right.
$$

takes the form (1.2).

Such a system of equations was studied by G.-H. Halphen [11], where, based on the knowledge of the first integrals, the author explicitly parameterized the two components of the impulse of the body-fluid system (in our notation $u_{1}$ and $u_{2}$ ) by Weierstrass elliptic functions and derived a differential equation on the remaining component $u_{3}$. Note that the above-mentioned equation describes the squared derivative $\dot{u}_{3}^{2}$, and even if solutions to the equation are found, one faces the problem of choosing the sign of the derivative when passing through critical points. In addition, the author provides explicit expressions for some rational function of $u_{3}$ and for the components $u_{4}, u_{5}$ of the angular momentum under the assumption that $u_{3}$ is known. Thus, in [11], the author obtained explicit formulas for individual components of the extremal control in Weierstrass elliptic functions. In the present work, we also present no explicit formulas of all components of the extremal control in the general case, however, we will derive a solution to the problem in operator form. The formulas obtained will allow us to construct an approximate analytical solution for small $u_{6}$. 
In the initial work [7], the problem was studied for $u_{6}=0$ under the assumption that there are no cusp points on the spacial projections of the geodesics, which is equivalent to $u_{3} \neq 0$. The assumption $u_{6}=0$ results in the minimum length among all possible trajectories connecting the boundary conditions $\left(q_{0}, N_{0}\right)$ and $\left(q_{1}, R_{v_{1}^{1}, \alpha} N_{1}\right)$, where $R_{v_{1}^{1}, \alpha}$ denotes the rotation by the angle $\alpha \in S^{1}$ around the vector $v_{1}^{1}$, see Fig. 1. In our previous work [12], we obtained explicit expressions for the extremal control (solution to system (1.2)) without any restrictions on the cusp points, but under the assumption $u_{6}=0$. Such trajectories (with $u_{6}=0$ ) are the most demanded for applications in image processing, see [7, Theorem 1]. However, in the theoretical aspect, the case $u_{6} \neq 0$ is of great interest as a general case of the model problem.

In this paper, we study the general case when the parameter $u_{6}$ is arbitrary. Based on the analysis of the Hamiltonian system of PMP, in Section 2 we find expressions of the extremal controls in operator form. Further, in Section 3 we study asymptotics of the extremal controls for $u_{6} \rightarrow 0$. The numerical simulations in Section 4 show a high accuracy of the formulas obtained.

\section{Expression of the extremal controls}

In this section, we derive from system (1.2) the expression of the extremal controls $u_{1}, u_{2}$, $u_{4}, u_{5}$ via $u_{3}$ in operator form. Let us introduce the functions

$$
v_{1}=u_{1}+u_{5}, \quad v_{2}=u_{2}+u_{4}, \quad v_{4}=u_{4}-u_{2}, \quad v_{5}=u_{5}-u_{1} .
$$

In this notation, the first, second, fourth, and fifth equations of system (1.2) are rewritten as

$$
\left\{\begin{array}{l}
\dot{v}_{1}=-u_{3} v_{1}+\left(v_{2}+v_{4}\right) \frac{u_{6}}{2}, \\
\dot{v}_{2}=u_{3} v_{2}-\left(v_{1}+v_{5}\right) \frac{u_{6}}{2}, \\
\dot{v}_{4}=-u_{3} v_{4}-\left(v_{1}+v_{5}\right) \frac{u_{6}}{2}, \\
\dot{v}_{5}=u_{3} v_{5}+\left(v_{2}+v_{4}\right) \frac{u_{6}}{2} .
\end{array}\right.
$$

From the equation $\dot{u}_{6}=0$ it is clear that $u_{6}$ is a constant. The further plan is to obtain the expressions of the functions $u_{1}, u_{2}, u_{4}, u_{5}$ in terms of $u_{3}$ by substituting them into the equation

$$
\dot{u}_{3}=u_{1} u_{5}-u_{2} u_{4}
$$

and find $u_{3}$ from it, and then all the other extremal controls.

Let a function $U$ be given by

$$
U(t)=\int_{0}^{t} u_{3}(\tau) \mathrm{d} \tau,
$$

and let us assume that $v_{1}(t)$ has the following form:

$$
v_{1}(t)=A(t) \mathrm{e}^{-U(t)} .
$$

To simplify notation in the further computations, we will often omit the dependence on $t$. Using (2.5) and system (2.2), we get

$$
\dot{v}_{1}=\dot{A} \mathrm{e}^{-U}-u_{3} A \mathrm{e}^{-U}=\dot{A} \mathrm{e}^{-U}-u_{3} v_{1},
$$




$$
\dot{v}_{1}+u_{3} v_{1}=\left(v_{2}+v_{4}\right) \frac{u_{6}}{2}=\dot{A} \mathrm{e}^{-U} \Rightarrow \dot{A}=u_{6}\left(\frac{v_{2}+v_{4}}{2}\right) \mathrm{e}^{U} .
$$

Integrating the last equation, we find the function $A(t)$ :

$$
A(t)=A(0)+u_{6} \int_{0}^{t} \frac{v_{2}(\tau)+v_{4}(\tau)}{2} \mathrm{e}^{U(\tau)} \mathrm{d} \tau
$$

Combining (2.4) and (2.5), we obtain $A(0)=v_{1}(0)$. Therefore, we have

$$
v_{1}(t)=v_{1}(0) \mathrm{e}^{-U(t)}+u_{6} \mathrm{e}^{-U(t)} \int_{0}^{t} \frac{v_{2}(\tau)+v_{4}(\tau)}{2} \mathrm{e}^{U(\tau)} \mathrm{d} \tau .
$$

Similarly, we obtain the expression for $v_{5}(t)$ :

$$
v_{5}(t)=v_{5}(0) \mathrm{e}^{U(t)}+u_{6} \mathrm{e}^{U(t)} \int_{0}^{t} \frac{v_{2}(\tau)+v_{4}(\tau)}{2} \mathrm{e}^{-U(\tau)} \mathrm{d} \tau .
$$

Summing (2.6) and (2.7), we get the equality

$$
v_{1}(t)+v_{5}(t)=v_{1}(0) \mathrm{e}^{-U(t)}+v_{5}(0) \mathrm{e}^{U(t)}+u_{6} \int_{0}^{t}\left(v_{2}(\tau)+v_{4}(\tau)\right) \operatorname{ch}(U(t)-U(\tau)) \mathrm{d} \tau .
$$

Further, we note that the second and third equations are related in the same way as the first and fourth equations in system (2.2), and differ only in sign. Therefore, we have the following expressions for $v_{2}(t), v_{4}(t)$ and their sum:

$$
\begin{gathered}
v_{2}(t)=v_{2}(0) \mathrm{e}^{U(t)}-u_{6} \mathrm{e}^{U(t)} \int_{0}^{t} \frac{v_{1}(\tau)+v_{5}(\tau)}{2} \mathrm{e}^{-U(\tau)} \mathrm{d} \tau, \\
v_{4}(t)=v_{4}(0) \mathrm{e}^{-U(t)}-u_{6} \mathrm{e}^{-U(t)} \int_{0}^{t} \frac{v_{1}(\tau)+v_{5}(\tau)}{2} \mathrm{e}^{U(\tau)} \mathrm{d} \tau, \\
v_{2}(t)+v_{4}(t)=v_{2}(0) \mathrm{e}^{U(t)}+v_{4}(0) \mathrm{e}^{-U(t)}-u_{6} \int_{0}^{t}\left(v_{1}(\tau)+v_{5}(\tau)\right) \operatorname{ch}(U(t)-U(\tau)) \mathrm{d} \tau .
\end{gathered}
$$

Substituting expressions (2.8) and (2.11) into (2.1), we see that the extremal controls $u_{4}(t)$ and $u_{5}(t)$ satisfy the system

$$
\left\{\begin{array}{l}
u_{4}(t)=\frac{u_{2}(0)+u_{4}(0)}{2} \mathrm{e}^{U(t)}+\frac{u_{4}(0)-u_{2}(0)}{2} \mathrm{e}^{-U(t)}-u_{6} \int_{0}^{t} u_{5}(\tau) \operatorname{ch}(U(t)-U(\tau)) \mathrm{d} \tau, \\
u_{5}(t)=\frac{u_{1}(0)+u_{5}(0)}{2} \mathrm{e}^{-U(t)}+\frac{u_{5}(0)-u_{1}(0)}{2} \mathrm{e}^{U(t)}+u_{6} \int_{0}^{t} u_{4}(\tau) \operatorname{ch}(U(t)-U(\tau)) \mathrm{d} \tau .
\end{array}\right.
$$


Let

$$
\begin{aligned}
& G(t)=\frac{u_{2}(0)+u_{4}(0)}{2} \mathrm{e}^{U(t)}+\frac{u_{4}(0)-u_{2}(0)}{2} \mathrm{e}^{-U(t)}, \\
& F(t)=\frac{u_{1}(0)+u_{5}(0)}{2} \mathrm{e}^{-U(t)}+\frac{u_{5}(0)-u_{1}(0)}{2} \mathrm{e}^{U(t)} .
\end{aligned}
$$

Let us introduce the operator

$$
L[f]=\int_{0}^{t} f(\tau) \operatorname{ch}(U(t)-U(\tau)) \mathrm{d} \tau .
$$

In this notation, system (2.12) takes the form

$$
\left\{\begin{array}{l}
u_{4}(t)=G(t)-u_{6} L\left[u_{5}\right], \\
u_{5}(t)=F(t)+u_{6} L\left[u_{4}\right] .
\end{array}\right.
$$

The first equation of system (2.13) and linearity of the operator $L$ imply the equality

$$
L\left[u_{4}\right]=L[G]-u_{6}^{2} L^{2}\left[u_{5}\right] .
$$

Substituting this expression into the second equation of system (2.13), we find

$$
u_{5}=F+u_{6} L[G]-u_{6}^{2} L^{2}\left[u_{5}\right] .
$$

In operator form, this equation is equivalent to the following:

$$
\left(I+u_{6}^{2} L^{2}\right)\left[u_{5}\right]=F+u_{6} L[G],
$$

where $I$ is the identity operator.

Now, using (2.14), we get the exact expression for the extremal control $u_{5}$ :

$$
u_{5}=\left(I+u_{6}^{2} L^{2}\right)^{-1}\left[F+u_{6} L[G]\right] .
$$

In the same manner, we can express $u_{4}$ :

$$
\begin{array}{r}
L\left[u_{5}\right]=L[F]+u_{6} L^{2}\left[u_{4}\right], \\
u_{4}=G-u_{6} L[F]-u_{6}^{2} L^{2}\left[u_{4}\right], \\
\left(I+u_{6}^{2} L^{2}\right)\left[u_{4}\right]=G-u_{6} L[F] .
\end{array}
$$

Thus we get the exact expression for the extremal control $u_{4}$ :

$$
u_{4}=\left(I+u_{6}^{2} L^{2}\right)^{-1}\left[G-u_{6} L[F]\right] .
$$

Let us substantiate the correctness of representations (2.15), (2.16). The operator $L$ is well defined in all spaces $\mathbb{L}[0, a], a>0$, of Lebesgue integrable functions $f$ on the interval $[0, a]$ with standard norm

$$
\|f\|_{\mathbb{L}[0, a]}:=\int_{0}^{a}|f(\tau)| \mathrm{d} \tau .
$$


It belongs to the class of integral operators of the form

$$
A[f](t)=\int_{0}^{t} f(\tau) K(\tau, t) \mathrm{d} \tau
$$

with continuous and symmetric kernel $K$. As is well known from the course of functional analysis [13], the norms

$$
\|A\|_{B L}:=\sup _{\|f\|_{\mathbb{L}[0, a]} \leqslant 1}\|A f\|_{\mathbb{L}[0, a]}
$$

of the powers of such operators rapidly tend to zero:

$$
\left\|A^{n}\right\|_{B L}=O\left(\frac{C^{n}}{n !}\right), \quad n \rightarrow \infty,
$$

where $C$ is a constant determined by the kernel $K$.

Therefore, the operator $I-k A$ is invertible for any $k \in \mathbb{R}$, and the inverse operator can be written in the form of a series $(I-k A)^{-1}=\sum_{n=0}^{\infty} k^{n} A^{n}$, converging by the operator norm in the space of linear operators, acting from $\mathbb{L}[0, a]$ to $\mathbb{L}[0, a]$. In our case, we have

$$
\left(I+u_{6}^{2} L^{2}\right)^{-1}=\sum_{n=0}^{\infty}(-1)^{n} u_{6}^{2 n} L^{2 n}=I-u_{6}^{2} L^{2}+u_{6}^{4} L^{4}-u_{6}^{6} L^{6}+\ldots
$$

Similarly, we can express $u_{1}(t), u_{2}(t)$. Using (2.1), (2.6), (2.7), (2.9), (2.10), we have

$$
\begin{aligned}
u_{1}(t)=\frac{v_{1}(t)-v_{5}(t)}{2}= & \frac{1}{2} v_{1}(0) \mathrm{e}^{-U(t)}-\frac{1}{2} v_{5}(0) \mathrm{e}^{U(t)}+ \\
& \frac{u_{6}}{2} \int_{0}^{t}\left(v_{2}(\tau)+v_{4}(\tau)\right) \frac{\mathrm{e}^{U(\tau)-U(t)}-\mathrm{e}^{U(t)-U(\tau)}}{2} \mathrm{~d} \tau, \\
u_{2}(t)=\frac{v_{2}(t)-v_{4}(t)}{2}= & \frac{1}{2} v_{2}(0) \mathrm{e}^{U(t)}-\frac{1}{2} v_{4}(0) \mathrm{e}^{-U(t)}- \\
& \frac{u_{6}}{2} \int_{0}^{t}\left(v_{1}(\tau)+v_{5}(\tau)\right) \frac{\mathrm{e}^{U(\tau)-U(t)}-\mathrm{e}^{U(t)-U(\tau)}}{2} \mathrm{~d} \tau .
\end{aligned}
$$

Simplifying these expressions, we get

$$
\begin{aligned}
& u_{1}(t)=\frac{u_{1}(0)+u_{5}(0)}{2} \mathrm{e}^{-U(t)}-\frac{u_{5}(0)-u_{1}(0)}{2} \mathrm{e}^{U(t)}-u_{6} \int_{0}^{t} u_{4}(\tau) \operatorname{sh}(U(t)-U(\tau)) \mathrm{d} \tau, \\
& u_{2}(t)=\frac{u_{2}(0)+u_{4}(0)}{2} \mathrm{e}^{U(t)}-\frac{u_{4}(0)-u_{2}(0)}{2} \mathrm{e}^{-U(t)}-u_{6} \int_{0}^{t} u_{5}(\tau) \operatorname{sh}(U(t)-U(\tau)) \mathrm{d} \tau .
\end{aligned}
$$

Let

$$
\begin{aligned}
& F_{1}(t)=\frac{u_{1}(0)+u_{5}(0)}{2} \mathrm{e}^{-U(t)}-\frac{u_{5}(0)-u_{1}(0)}{2} \mathrm{e}^{U(t)}, \\
& G_{1}(t)=\frac{u_{2}(0)+u_{4}(0)}{2} \mathrm{e}^{U(t)}-\frac{u_{4}(0)-u_{2}(0)}{2} \mathrm{e}^{-U(t)} .
\end{aligned}
$$


Let us introduce the operator

$$
M[f]=\int_{0}^{t} f(\tau) \operatorname{sh}(U(t)-U(\tau)) \mathrm{d} \tau .
$$

In this notation, Eqs. (2.17) take the form

$$
\left\{\begin{array}{l}
u_{1}(t)=F_{1}(t)-u_{6} M\left[u_{4}\right], \\
u_{2}(t)=G_{1}(t)-u_{6} M\left[u_{5}\right] .
\end{array}\right.
$$

Note that the operators $L$ and $M$ commute and the following relations hold:

$$
\begin{aligned}
& M\left[u_{4}\right]=\left(I+u_{6}^{2} L^{2}\right)^{-1}\left[M[G]-u_{6} M L[F]\right], \\
& M\left[u_{5}\right]=\left(I+u_{6}^{2} L^{2}\right)^{-1}\left[M[F]+u_{6} M L[G]\right] .
\end{aligned}
$$

Finally, substituting the expressions obtained for $u_{1}, u_{2}, u_{4}, u_{5}$ into Eq. (2.3), we get a Cauchy problem with the second-order integro-differential operator equation for the remaining unknown function $U(t)$ :

$$
\ddot{U}(t)=u_{1}(t) u_{5}(t)-u_{2}(t) u_{4}(t), \quad U(0)=0, \quad \dot{U}(0)=u_{3}(0) .
$$

Summing up, we obtain the following theorem.

Theorem 1. Extremal controls in the sub-Riemannian problem on the group of motions of three-dimensional Euclidean space have the form

$$
\begin{aligned}
& u_{1}(t)=F_{1}-u_{6}\left(I+u_{6}^{2} L^{2}\right)^{-1}\left[M[G]-u_{6} M L[F]\right], \\
& u_{2}(t)=G_{1}-u_{6}\left(I+u_{6}^{2} L^{2}\right)^{-1}\left[M[F]+u_{6} M L[G]\right], \\
& u_{3}(t)=\dot{U}(t), \\
& u_{4}(t)=\left(I+u_{6}^{2} L^{2}\right)^{-1}\left[G-u_{6} L[F]\right], \\
& u_{5}(t)=\left(I+u_{6}^{2} L^{2}\right)^{-1}\left[F+u_{6} L[G]\right],
\end{aligned}
$$

where

$$
\begin{aligned}
& F_{1}=F_{1}(t)=\frac{u_{1}(0)+u_{5}(0)}{2} \mathrm{e}^{-U(t)}-\frac{u_{5}(0)-u_{1}(0)}{2} \mathrm{e}^{U(t)}, \\
& G_{1}=G_{1}(t)=\frac{u_{2}(0)+u_{4}(0)}{2} \mathrm{e}^{U(t)}-\frac{u_{4}(0)-u_{2}(0)}{2} \mathrm{e}^{-U(t)}, \\
& F=F(t)=\frac{u_{1}(0)+u_{5}(0)}{2} \mathrm{e}^{-U(t)}+\frac{u_{5}(0)-u_{1}(0)}{2} \mathrm{e}^{U(t)}, \\
& G=G(t)=\frac{u_{2}(0)+u_{4}(0)}{2} \mathrm{e}^{U(t)}+\frac{u_{4}(0)-u_{2}(0)}{2} \mathrm{e}^{-U(t)}, \\
& L[f]=L[f](t)=\int_{0}^{t} f(\tau) \operatorname{ch}(U(t)-U(\tau)) d \tau, \\
& M[f]=M[f](t)=\int_{0}^{t} f(\tau) \operatorname{sh}(U(t)-U(\tau)) d \tau,
\end{aligned}
$$

and the function $U(t)$ satisfies the Cauchy problem

$$
\ddot{U}(t)=u_{1}(t) u_{5}(t)-u_{2}(t) u_{4}(t), \quad U(0)=0, \quad \dot{U}(0)=u_{3}(0) .
$$




\section{Asymptotics of extremal controls near $u_{6}=0$}

Let us consider the case when the parameter $u_{6}$ is close to zero. We aim to obtain the asymptotics of the formulas in Theorem 1. Using (2.15), (2.16) and (2.18), we find

$$
\begin{aligned}
& u_{1}=F_{1}-u_{6} M[G]+O\left(u_{6}^{2}\right), \\
& u_{2}=G_{1}-u_{6} M[F]+O\left(u_{6}^{2}\right), \\
& u_{4}=G-u_{6} L[F]+O\left(u_{6}^{2}\right), \\
& u_{5}=F+u_{6} L[G]+O\left(u_{6}^{2}\right) .
\end{aligned}
$$

Up to $O\left(u_{6}^{2}\right)$, the expression $u_{1} u_{5}-u_{2} u_{4}$ is approximately equal to

$$
\begin{aligned}
& \left(F_{1}-u_{6} M[G]\right)\left(F+u_{6} M[F]\right)-\left(G_{1}-u_{6} M[F]\right)\left(G-u_{6} L[F]\right) \\
\approx & F_{1} F+u_{6}\left(F_{1} L[G]-F M[G]\right)-G G_{1}+u_{6}\left(G_{1} L[F]+G M[F]\right),
\end{aligned}
$$

and, up to $O\left(u_{6}^{2}\right)$, Eq. (2.19) takes the form

$$
\ddot{U}=F_{1} F-G G_{1}+u_{6}\left(F_{1} L[G]-F M[G]+G_{1} L[F]+G M[F]\right) .
$$

Let

$$
\alpha=\frac{u_{2}(0)+u_{4}(0)}{2}, \quad \beta=\frac{u_{4}(0)-u_{2}(0)}{2}, \quad \tilde{\alpha}=\frac{u_{1}(0)+u_{5}(0)}{2}, \quad \tilde{\beta}=\frac{u_{5}(0)-u_{1}(0)}{2} .
$$

Then, by definition of $G, F, G_{1}$ and $F_{1}$, we have

$$
\begin{array}{rlrl}
G(t) & =\alpha \mathrm{e}^{U(t)}+\beta \mathrm{e}^{-U(t)}, & F(t) & =\tilde{\alpha} \mathrm{e}^{-U(t)}+\tilde{\beta} \mathrm{e}^{U(t)}, \\
G_{1}(t) & =\alpha \mathrm{e}^{U(t)}-\beta \mathrm{e}^{-U(t)}, & F_{1}(t)=\tilde{\alpha} \mathrm{e}^{-U(t)}-\tilde{\beta} \mathrm{e}^{U(t)},
\end{array}
$$

Hence, we find that the main term of the right-hand side of Eq. (3.2) is equal to

$$
F F_{1}-G G_{1}=A \mathrm{e}^{-2 U}-B \mathrm{e}^{2 U},
$$

where $A=\tilde{\alpha}^{2}+\beta^{2}, B=\tilde{\beta}^{2}+\alpha^{2}$.

Next, let us compute the operators $L[G], L[F], M[G], M[F]$ :

$$
\begin{aligned}
& L[G](t)=\frac{t}{2} G(t)+\frac{\alpha}{2} \mathrm{e}^{-U(t)} \int_{0}^{t} \mathrm{e}^{2 U(\tau)} d \tau+\frac{\beta}{2} \mathrm{e}^{U(t)} \int_{0}^{t} \mathrm{e}^{-2 U(\tau)} d \tau, \\
& M[G](t)=\frac{t}{2} G_{1}(t)-\frac{\alpha}{2} \mathrm{e}^{-U(t)} \int_{0}^{t} \mathrm{e}^{2 U(\tau)} d \tau+\frac{\beta}{2} \mathrm{e}^{U(t)} \int_{0}^{t} \mathrm{e}^{-2 U(\tau)} d \tau, \\
& L[F](t)=\frac{t}{2} F(t)+\frac{\tilde{\alpha}}{2} \mathrm{e}^{U(t)} \int_{0}^{t} \mathrm{e}^{-2 U(\tau)} d \tau+\frac{\tilde{\beta}}{2} \mathrm{e}^{-U(t)} \int_{0}^{t} \mathrm{e}^{2 U(\tau)} d \tau, \\
& M[F](t)=\frac{t}{2} F_{1}(t)+\frac{\tilde{\alpha}}{2} \mathrm{e}^{U(t)} \int_{0}^{t} \mathrm{e}^{-2 U(\tau)} d \tau-\frac{\tilde{\beta}}{2} \mathrm{e}^{-U(t)} \int_{0}^{t} \mathrm{e}^{2 U(\tau)} d \tau .
\end{aligned}
$$


Therefore, the coefficient in front of $u_{6}$ on the right-hand side of (3.2) is expressed as follows:

$$
\begin{gathered}
F_{1}(t) L[G](t)-F(t) M[G](t)+G_{1}(t) L[F](t)+G(t) M[F](t)= \\
(\alpha \tilde{\alpha}-\beta \tilde{\beta})\left(t+2 \int_{0}^{t} \operatorname{ch}(2(U(t)-U(\tau))) d \tau\right)-t\left(\alpha \tilde{\beta} \mathrm{e}^{2 U(t)}+\tilde{\alpha} \beta \mathrm{e}^{-2 U(t)}\right) .
\end{gathered}
$$

Summing up, we obtain the following statement.

Theorem 2. Let $u_{6}$ be close to zero. The extremal controls in the sub-Riemannian problem on the group of motions of three-dimensional Euclidean space are approximately expressed up to $O\left(u_{6}^{2}\right)$ as follows:

$$
\begin{aligned}
& u_{1}(t)=F_{1}(t)-u_{6} M[G](t), \\
& u_{2}(t)=G_{1}(t)-u_{6} M[F](t), \\
& u_{3}(t)=\dot{U}(t), \\
& u_{4}(t)=G(t)-u_{6} L[F](t), \\
& u_{5}(t)=F(t)+u_{6} L[G](t),
\end{aligned}
$$

where

$$
\begin{aligned}
& F_{1}(t)=\tilde{\alpha} \mathrm{e}^{-U(t)}-\tilde{\beta} \mathrm{e}^{U(t)}, \quad G_{1}(t)=\alpha \mathrm{e}^{U(t)}-\beta \mathrm{e}^{-U(t)}, \\
& F(t)=\tilde{\alpha} \mathrm{e}^{-U(t)}+\tilde{\beta} \mathrm{e}^{U(t)}, \quad G(t)=\alpha \mathrm{e}^{U(t)}+\beta \mathrm{e}^{-U(t)}, \\
& L[f](t)=\int_{0}^{t} f(\tau) \operatorname{ch}(U(t)-U(\tau)) d \tau \\
& M[f](t)=\int_{0}^{t} f(\tau) \operatorname{sh}(U(t)-U(\tau)) d \tau \\
& \alpha=\frac{u_{2}(0)+u_{4}(0)}{2}, \quad \beta=\frac{u_{4}(0)-u_{2}(0)}{2} \\
& \tilde{\alpha}=\frac{u_{1}(0)+u_{5}(0)}{2}, \quad \tilde{\beta}=\frac{u_{5}(0)-u_{1}(0)}{2}
\end{aligned}
$$

and the function $U(t)$ satisfies the Cauchy problem

$$
\left\{\begin{array}{l}
\ddot{U}(t)=f_{0}(U(t))+u_{6}\left(t f_{1}(U(t))+k \int_{0}^{t} \operatorname{ch}(2 U(t)-2 U(\tau)) d \tau\right), \\
U(0)=0, \quad \dot{U}(0)=u_{3}(0)
\end{array}\right.
$$

where $k=\alpha \tilde{\alpha}-\beta \tilde{\beta}$,

$$
\begin{aligned}
& f_{0}(U)=\left(\tilde{\alpha}^{2}+\beta^{2}\right) \mathrm{e}^{-2 U}-\left(\tilde{\beta}^{2}+\alpha^{2}\right) \mathrm{e}^{2 U}, \\
& f_{1}(U)=k-\alpha \tilde{\beta} \mathrm{e}^{2 U}-\tilde{\alpha} \beta \mathrm{e}^{-2 U} .
\end{aligned}
$$

When numerically solving the Cauchy problem for the integro-differential equation (3.3), some difficulties arise; an ordinary differential equation is preferable for the numerical methods. In this regard, we prove the following theorem. 
Theorem 3. Any solution to the integro-differential equation (3.3) satisfies the fourthorder ordinary differential equation

$$
\dot{U} U^{(4)}=\dddot{U} \ddot{U}+4 \ddot{U} \dot{U}^{3}-u_{6} \ddot{U} f_{1}(U)-4 u_{6} k t \dot{U}^{3}+2 u_{6} f_{1}^{\prime}(U) \dot{U}^{2} .
$$

Proof. Consider the function in (3.3)

$$
Y(t)=\int_{0}^{t} \operatorname{ch}(2 U(t)-2 U(\tau)) d \tau
$$

and compute its first- and second-order derivatives by $t$ :

$$
\begin{aligned}
\dot{Y}(t) & =1+2 \dot{U}(t) \int_{0}^{t} \operatorname{sh}(2 U(t)-2 U(\tau)) d \tau \\
\ddot{Y}(t) & =2\left(\ddot{U}(t) \int_{0}^{t} \operatorname{sh}(2 U(t)-2 U(\tau)) d \tau+\dot{U}(t) \frac{d}{d t}\left(\int_{0}^{t} \operatorname{sh}(2 U(t)-2 U(\tau)) d \tau\right)\right) \\
& =\ddot{U}(t) \frac{\dot{Y}(t)-1}{\dot{U}(t)}+4 \dot{U}^{2}(t) Y(t) .
\end{aligned}
$$

Using (3.3), we have

$$
u_{6} k Y(t)=\ddot{U}(t)-f_{0}(U(t))-u_{6} t f_{1}(U(t)) .
$$

Differentiating equality (3.8) by $t$, we get

$$
\begin{aligned}
u_{6} k \dot{Y}(t) & =\dddot{U}(t)-\dot{U}(t) f_{0}^{\prime}(U(t))-u_{6} f_{1}(U(t))-u_{6} t \dot{U}(t) f_{1}^{\prime}(U(t)), \\
u_{6} k \ddot{Y}(t) & =U^{(4)}(t)-\ddot{U}(t) f_{0}^{\prime}(U(t))-\dot{U}^{2}(t) f_{0}^{\prime \prime}(U(t))-2 u_{6} \dot{U}(t) f_{1}^{\prime}(U(t))- \\
& -u_{6} t \dot{U}^{2}(t) f_{1}^{\prime \prime}(U(t))-u_{6} t \ddot{U}(t) f_{1}^{\prime}(U(t)) .
\end{aligned}
$$

Substituting expressions (3.7) of $\ddot{Y}$ via $\dot{Y}, \dot{U}, \ddot{U}$ into the left-hand side of (3.10), we obtain the following equation:

$$
\begin{aligned}
\frac{u_{6} k \ddot{U}(t)}{\dot{U}(t)}(\dot{Y}(t)-1) & +4 u_{6} k \dot{U}^{2}(t) Y(t)=U^{(4)}(t)-\ddot{U}(t) f_{0}^{\prime}(U(t))-\dot{U}^{2}(t) f_{0}^{\prime \prime}(U(t))- \\
& -2 u_{6} \dot{U}(t) f_{1}^{\prime}(U(t))-u_{6} t \dot{U}^{2}(t) f_{1}^{\prime \prime}(U(t))-u_{6} t \ddot{U}(t) f_{1}^{\prime}(U(t)) .
\end{aligned}
$$

Let us express $\dot{Y}$ and $Y$ on the left-hand side of Eq. (3.11) in terms of the function $U$ and its derivatives according to relations (3.8) and (3.9). Omitting the argument $t$ of the function $U$ for simplicity of notation, we obtain the equation

$$
\begin{aligned}
U^{(4)} & =\frac{\dddot{U} \ddot{U}}{\dot{U}}+\ddot{U}\left(4 \dot{U}^{2}-u_{6} \frac{f_{1}(U)}{\dot{U}}\right)+2 U_{6} \dot{U} f_{1}^{\prime}(U)+ \\
& +\dot{U}^{2}\left(f_{0}^{\prime \prime}(U)+u_{6} t f_{1}^{\prime \prime}(U)-4 f_{0}(U)-4 u_{6} t f_{1}(U)\right) .
\end{aligned}
$$

This equation can be simplified due to the identities

$$
f_{0}^{\prime \prime}(U) \equiv 4 f_{0}(U), \quad f_{1}^{\prime \prime}(U)=4\left(f_{1}(U)-k\right) .
$$

Applying these identities to (3.12) and multiplying both sides of (3.12) by $\dot{U}$, we obtain Eq. (3.6). The theorem is proved. 

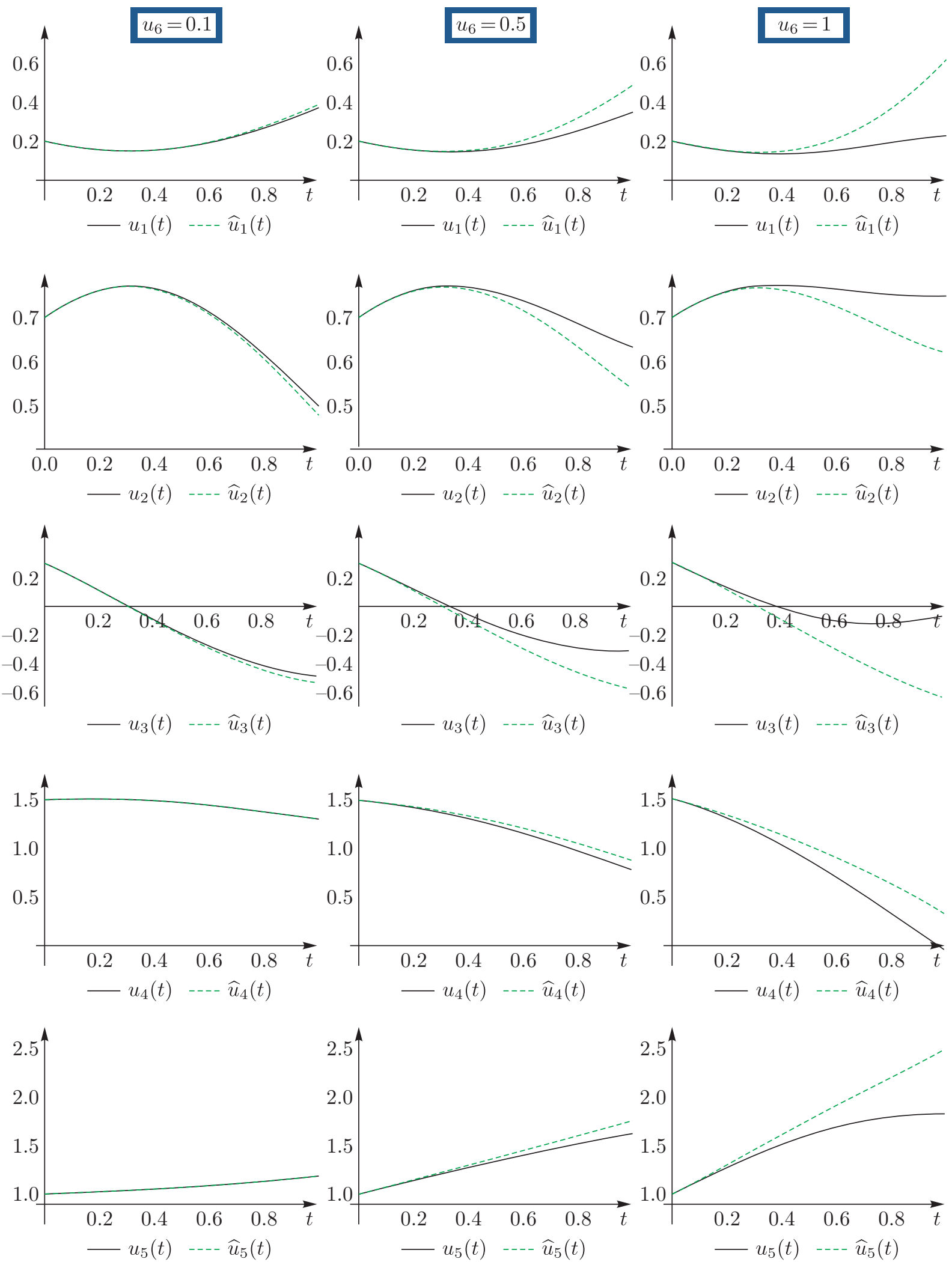

Fig. 2. Comparison of the graphs of the extremal controls $u_{1}, u_{2}, u_{3}, u_{4}, u_{5}$ (by rows) for $u_{6} \in\{0.1,0.5,1\}$ (by columns) in the sub-Riemannian problem on $\mathrm{SE}_{3}$, computed by the approximate formulas in Theorem 2 (dashed lines) and by integrating system (1.2), which determines the exact solutions (solid lines). 


\section{Numerical simulations}

In this section, we present the results of numerical simulations evaluating the accuracy of the approximation for the solutions of system (1.2) by the expressions in Theorem 2.

In Fig. 2, we compare the graphs of the extremal controls with their approximate expressions in Theorem 2 with initial values $u_{1}(0)=0.2, u_{2}(0)=0.7, u_{3}(0)=0.3, u_{4}(0)=1.5, u_{5}(0)=1$ for $u_{6} \in\{0.1,0.5,1\}$. One can observe that, as expected, the asymptotic formulas in Theorem 2 are local. That is, they give a good approximation for a sufficiently short period of time $t \in[0, T]$, and the accuracy of the approximation increases when the value of $\left|u_{6}\right|$ decreases.

The numerical simulations are performed using Wolfram Mathematica. To solve the system of differential equations (1.2), we use the function NDSolve with standard options. The complete Wolfram Demonstration is available at: http://control.botik.ru/?page_id=2754.

\section{Conclusion}

In this paper, we study the problem of finding the extremal controls in the sub-Riemannian problem on the group of motions of three-dimensional Euclidean space $\mathrm{SE}_{3}$. Here, we consider the problem near $u_{6}=0$ and generalize the results of $[7,12]$. The main contributions are

- in Theorem 1, we present the solution in operator form for $u_{6} \in \mathbb{R}$;

- in Theorem 2, we derive the asymptotic formulas for the extremal controls in the case when $\left|u_{6}\right|$ is small;

- in Theorem 3, we reduce the integro-differential equation for the extremal control $u_{3}$ to the ordinary differential equation.

The practical applicability of the formulas obtained has been justified by the results of numerical simulations in Section 4, where we have confirmed that the solutions to the approximate system in Theorem 2 are close to the solutions found by integration of the original system (1.2) for small values of the parameter $\left|u_{6}\right|$ on a sufficiently small time interval.

The authors thank Prof. A.V. Tsiganov for pointing out the mechanical interpretation of the problem and for insightful correspondence.

\section{References}

[1] Jamieson, J. and Holderbaum, W., Trajectory Generation Using Sub-Riemannian Curves for Quadrotor UAVs, in Proc. of the European Control Conference (Linz, Austria, 2015), pp. $1645-1650$.

[2] Biggs, J. and Holderbaum, W., Optimal Kinematic Control of an Autonomous Underwater Vehicle, IEEE Trans. Automat. Control, 2009, vol. 54, no. 7, pp. 1623-1626.

[3] Duits, R., Dela Haije, T. C. J., Creusen, E. J., and Ghosh, A., Morphological and Linear Scale Spaces for Fiber Enhancement in DW-MRI, J. Math. Imaging Vision, 2013, vol. 46, no. 3, pp. 326-368.

[4] Bizyaev, I. A., Borisov, A. V., Kilin, A. A., and Mamaev, I. S., Integrability and Nonintegrability of Sub-Riemannian Geodesic Flows on Carnot Groups, Regul. Chaotic Dyn., 2016, vol.21, no.6, pp. 759-774.

[5] Lokutsievskii, L. V. and Sachkov, Yu. L., On the Liouville Integrability of Sub-Riemannian Problems on Carnot Groups of Step 4 and Higher, Sb. Math., 2018, vol.209, no. 5, pp.672-713; see also: Mat. Sb., 2018, vol. 209, no. 5, pp. 74-119. 
[6] Mashtakov, A.P. and Sachkov, Yu. L., Superintegrability of Left-Invariant Sub-Riemannian Structures on Unimodular Three-Dimensional Lie Groups, Differ. Equ., 2015, vol.51, no. 11, pp. 14761483; see also: Differ. Uravn., 2015, vol.51, no.11, pp. 1482-1488.

[7] Duits, R., Ghosh, A., Dela Haije, T.C.J., and Mashtakov, A., On Sub-Riemannian Geodesics in $S E(3)$ Whose Spatial Projections Do Not Have Cusps, J. Dyn. Control Syst., 2016, vol. 22, no. 4, pp. 771-805.

[8] Borisov, A. V. and Mamaev, I. S., Rigid Body Dynamics: Hamiltonian Methods, Integrability, Chaos, Izhevsk: R\&C Dynamics, Institute of Computer Science, 2005 (Russian).

[9] Kirchhoff, G., Vorlesungen über mathematische Physik: Vol. 1. Mechanik, Leipzig: Teubner, 1876.

[10] Borisov, A. V., Mamaev, I.S., and Bizyaev, I. A., Dynamical Systems with Non-Integrable Constraints: Vaconomic Mechanics, Sub-Riemannian Geometry, and Non-Holonomic Mechanics, Russian Math. Surveys, 2017, vol.72, no.5, pp.783-840; see also: Uspekhi Mat. Nauk, 2017, vol.72, no. 5(437), pp. 3-62.

[11] Halphen, G.-H., Sur le mouvement d'un solide dans un liquide, J. Math. Pures Appl. (4), 1888, vol. 4, pp. 5-82.

[12] Mashtakov, A.P. and Popov, A. Yu., Extremal Controls in the Sub-Riemannian Problem on the Group of Motions of Euclidean Space, Regul. Chaotic Dyn., 2017, vol. 22, no. 8, pp. 952-957.

[13] Kolmogorov, A. N. and Fomin, S. V., Elements of the Theory of Functions and Functional Analysis, New York: Dover, 1999. 